CLINICAL STUDY

\title{
Investigation of prolactin-related vasoinhibin in sera from patients with diabetic retinopathy
}

\author{
Jakob Triebel, Michael Huefner ${ }^{1}$ and Giuliano Ramadori \\ Department of Gastroenterology and Endocrinology, Center of Internal Medicine, Georg-August-University, Robert-Koch-Straße 40, 37075 Göttingen, \\ Germany and ${ }^{1}$ Endokrinologikum Göttingen, Von-Siebold-Straße 3, Göttingen, Germany \\ (Correspondence should be addressed to J Triebel; Email: jakob.triebel@gmx.de)
}

\begin{abstract}
Objective: In vitro experiments and in vivo studies on rodents demonstrate that N-terminal 14, 15, 16, 17 , and $18 \mathrm{kDa}$ fragments prolactin-related vasoinhibin (PRL-V) of human PRL are natural inhibitors of neovascularization in the retina and elsewhere. These N-terminal PRL fragments belong to a family of peptides named vasoinhibins, which act as endogenous regulators of angiogenesis and vascular function. These observations led to the hypothesis that PRL-V could play a role in the pathophysiology of diabetic retinopathy in humans. The purpose of this study was to investigate whether patients with diabetes mellitus and diabetic retinopathy have aberrant concentrations of PRL-V in the circulating blood.

Research design: We performed a case-control study and developed a new technique to semiquantitatively determine PRL-V in serum samples from 48 male subjects. The case group consisted of 21 patients with diabetes mellitus and proliferative or non-proliferative diabetic retinopathy. The control group consisted of 27 healthy subjects with no history of diabetes mellitus.

Methods: For the detection of PRL-V, we developed a new analytical method, consisting of immunologic and laser-induced fluorescence techniques.

Results: The case group had significantly lower PRL-V serum concentrations than the control group $(P=0.041)$. There was no significant difference between patients with proliferative and those with non-proliferative diabetic retinopathy.

Conclusion: We conclude that given the antiangiogenic and antivasopermeability actions of PRL-V, the decreased serum levels of PRL-V in patients with diabetes mellitus could contribute to the development and progression of diabetic retinopathy.
\end{abstract}

European Journal of Endocrinology $161345-353$

\section{Introduction}

Diabetic retinopathy is a common microvascular complication in patients with diabetes mellitus. Whereas early, non-proliferative stages of diabetic retinopathy are characterized by microaneurysms, hemorrhages, intraretinal microvascular abnormalities, and other pathological processes, the major characteristic of more advanced proliferative stages is neovascularization in the retina. Despite the identification of associated factors, such as chronic hyperglycemia (1), the underlying pathophysiological mechanisms leading to the development and progression of diabetic retinopathy are not fully understood (2).

Research in this field has revealed that besides its production and systemic release by the pituitary gland, prolactin (PRL) is also locally produced in multiple human tissues such as endothelial cells (3), where it acts as a cytokine (4). Studies revealed that full-length
PRL is proteolytically cleaved to various $\mathrm{N}$ - and C-terminal fragments $(5,6)$. Further investigations showed that the $\mathrm{N}$-terminal, in contrast to the C-terminal fragments (7), referred to as PRLrelated vasoinhibin (PRL-V), have antiangiogenic properties and belong to a family of antiangiogenic peptides that are also derived from $\mathrm{GH}$ and placental lactogen $(8,9)$. Based upon their functional and structural similarities, these peptides from different sources are classified as vasoinhibins and are characterized as endogenous regulators of angiogenesis and vascular function (10).

In vitro experiments and in vivo studies in rodents revealed that PRL-Vs inhibit neovascularization by several mechanisms (11), such as apoptosis-mediated vascular regression, thus being a potent inhibitor of angiogenesis in the retina (12) and elsewhere. In addition, PRL-V can inhibit vascular endothelial growth factor (VEGF)-induced endothelial cell proliferation (8) and VEGF-induced vasopermeability in the retinal 
vessels of diabetic rats (13), both believed to be important factors in the pathogenesis of diabetic retinopathy. These observations led to the hypothesis that PRL-V could be involved in the development and progression of diabetic retinopathy in humans $(10,11,14)$.

We performed a case-control study to investigate whether the serum levels of PRL-V correlate with the presence of diabetic retinopathy. Additionally, we report on the development of a highly sensitive and semi-quantitative method to detect PRL-V in human serum.

\section{Subjects and methods}

\section{General technical aspects}

For the detection of PRL-V and the systematic screening of the samples, we used a combination of immunologic and laser-induced fluorescence techniques. During this procedure, protein samples obtained by immunoprecipitation are loaded on a commercial, microfluidic based lab-on-a-chip device. Electrokinetic forces move the samples through a network of microchannels containing a gel/dye mixture, which function as a protein separation matrix with a resolution comparable with a 4-20\% gradient gel. The dye intercalates directly with the protein SDS-micelles and generates fluorescence, which is detected by a red laser. The amount of the protein is determined by the detection of the intensity of the fluorescence and the corresponding molecular weights are determined by an automated comparison with an internal standard.

\section{Immunoprecipitation}

One hundred and fifty microliter serum and $400 \mu \mathrm{l}$ PBS containing protease inhibitors (Cat. No.: 04693124001, Roche) was incubated with $50 \mu \mathrm{l}$ protein A-agarose (Cat. No.: 11134515001, Roche) for $1 \mathrm{~h}$ at $4{ }^{\circ} \mathrm{C}$ under rotation. The sample was centrifuged (centrifuge 5415R with rotor F45-24-11, Eppendorf, Hamburg, Germany) for $30 \mathrm{~s}$ at $3800 \boldsymbol{g}$ and $15 \mu \mathrm{l}$ mouse MAB 5602 against hPRL (Cat. No.: CAN-hPRL-4100-12, Diagnostics Biochem Canada, London, Canada) was added to the supernatant. After incubation for $6 \mathrm{~h}$ at $4{ }^{\circ} \mathrm{C}$ under rotation, the sample was incubated with $50 \mu \mathrm{l}$ protein G-agarose (Cat. No.: 11719416001 , Roche) for $16 \mathrm{~h}$ under the same conditions. The sample was centrifuged for $5 \mathrm{~min}$ at $3800 \boldsymbol{g}$ and the supernatant was discharged. The pellet was washed with PBS and then resuspended in $25 \mu \mathrm{l}$ PBS. Six microliter of the suspension was analyzed with the Bioanalyzer 2100 (Agilent Technologies, Waldbronn, Germany).

\section{Bioanalyzer 2100}

Adhering to the manufacturer's instructions for the protein 80 kit (Cat. No.: 5067-1515, Agilent Technologies), but with slight modifications, $6 \mu$ l of the sample was combined with $2 \mu$ d denaturation solution from the kit containing $3.5 \mathrm{v} / \mathrm{v} \% \beta$-mercaptoethanol. The vial was placed on a heating block for $5 \mathrm{~min}$ at $95{ }^{\circ} \mathrm{C}$. Six microliter of the sample was loaded on the chip (Cat. No. 5067-1515, Agilent Technologies) and underwent analysis by the device.
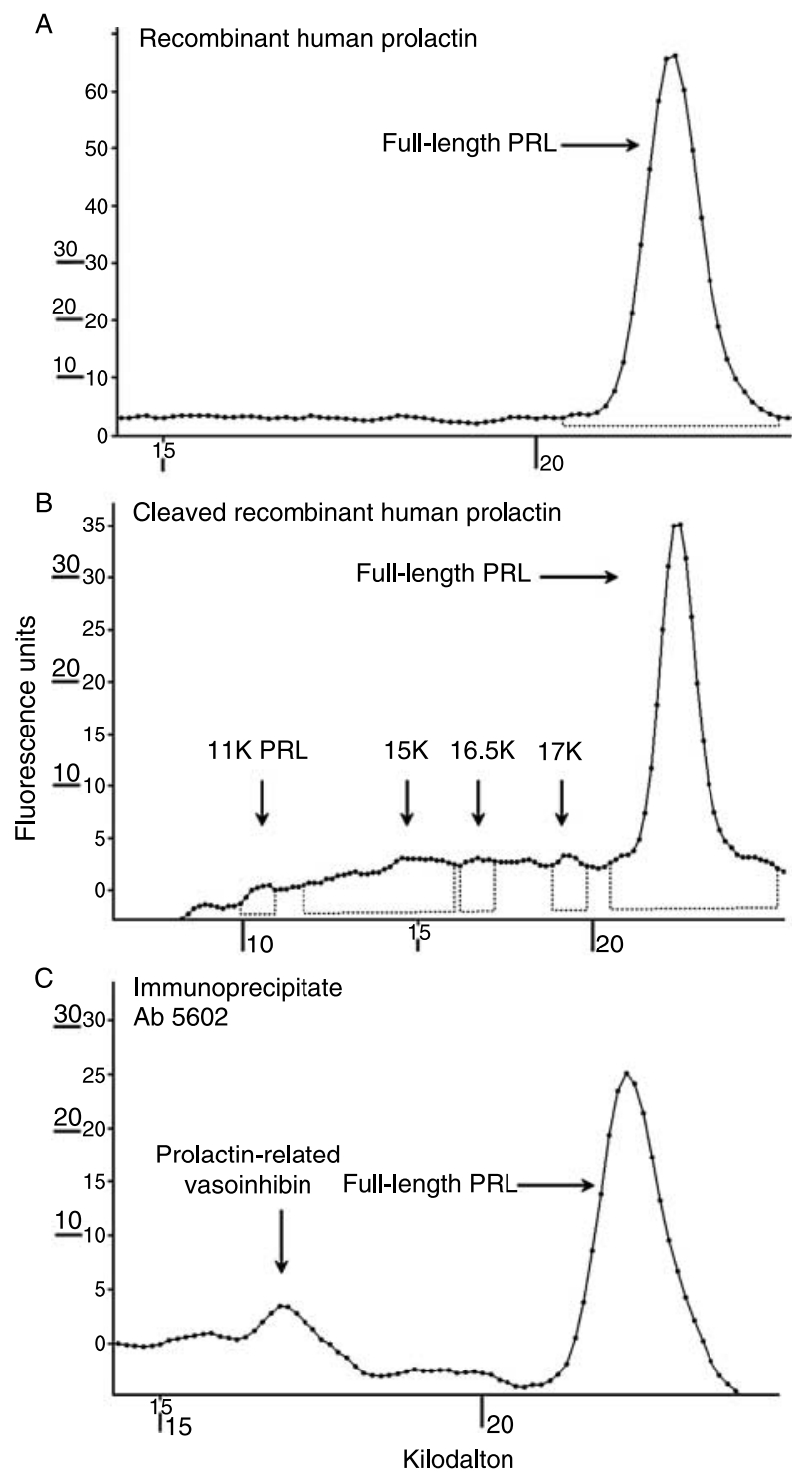

Figure 1 (A) Positive control. Five nanogram recombinant human PRL (rhPRL). (B) Five hundred nanogram rhPRL was incubated with $10 \mathrm{ng}$ cathepsin $\mathrm{D}$ from human liver in $100 \mu \mathrm{l}$ citrate-buffer at $\mathrm{pH} 3$ and $37^{\circ} \mathrm{C}$ for $1 \mathrm{~h}$. Analysis of $4 \mu$ diluted in $8 \mu \mathrm{l}$ sample buffer shows proteolytic cleavage of full-length $P R L$ into $P R L$ fragments. (C) Analysis of a serum-sample, immunoprecipitated with the antihPRL mouse MAB 5602 shows prolactin-related vasoinhibin - and 23 kDa-PRL signals. 


\section{Positive controls}

For positive controls, we used recombinant human PRL (rhPRL) from Escherichia Coli (Cat. No.: 40-267, NatuTec, Frankfurt, Germany). Five nanogram rhPRL was loaded on the chip and was analyzed by the device (Fig. 1A). Proteolysis of full-length PRL to the N-terminal PRL-fragments was conducted according the method of Piwnica et al. (6). Ten nanogram cathepsin D from human liver (Cat. No.: C869-25UG, Sigma) was incubated with $500 \mathrm{ng}$ rhPRL in $100 \mu \mathrm{l}$ citrate-buffer at $\mathrm{pH} 3$ and $37^{\circ} \mathrm{C}$ for $1 \mathrm{~h}$. The reaction was terminated by adding $100 \mu$ l SDS-PAGE sample buffer. Four microliter diluted in $8 \mu \mathrm{l}$ sample buffer and denaturation solution underwent analysis with the Bioanalyzer 2100 (Fig. 1B). The immunoprecipitation of a serum sample was also conducted with another mouse MAB, specific to a different epitope on hPRL (SC-80303, Santa Cruz Biotechnologies, Santa Cruz, CA, USA).

\section{Negative control}

For the negative control, we conducted the immunoprecipitation with a mouse MAB (Ab 5601) against hPRL with an epitope at the C-terminal end of PRL (Cat. No.: CAN-hPRL-4100-11, Diagnostics Biochem Canada). This antibody is specific to a C-terminal epitope (amino acids 150-199 of full length hPRL), which is not present on the N-terminal 16K PRL fragment. Consequently, the antibody binds to fulllength but not to N-terminal PRL fragments.

\section{Non-reducing conditions}

Immunoprecipitation and subsequent analysis of the sample with the Bioanalyzer 2100 under nonreducing conditions were conducted without the addition of $\beta$-mercaptoethanol or any other reducing agent (Fig. 2C).

\section{Electro-chemiluminescence immunoassay}

In addition to the determination of PRL in our assay, the concentration of full-length PRL was measured with a commercial electro-chemiluminescence immunoassay (ECLIA; Roche Diagnostics, Device: Cobas Modular Analytics E170, kit: PRL II, Cat. No.: 03203093 190). The sensitivity of the assay was $0.047 \mathrm{ng} / \mathrm{ml}$.

\section{Western blot analysis}

One hundred microliter of serum was incubated with $450 \mu \mathrm{l}$ PBS containing protease inhibitors (Roche) and $50 \mu \mathrm{l}$ protein A-agarose (Roche) for $1 \mathrm{~h}$ at $4{ }^{\circ} \mathrm{C}$ under rotation. The sample was centrifuged for $20 \mathrm{~s}$ at 6000 r.p.m. and $10 \mu \mathrm{l}$ rabbit polyclonal antibody against human PRL (A0569, DakoCytomation,

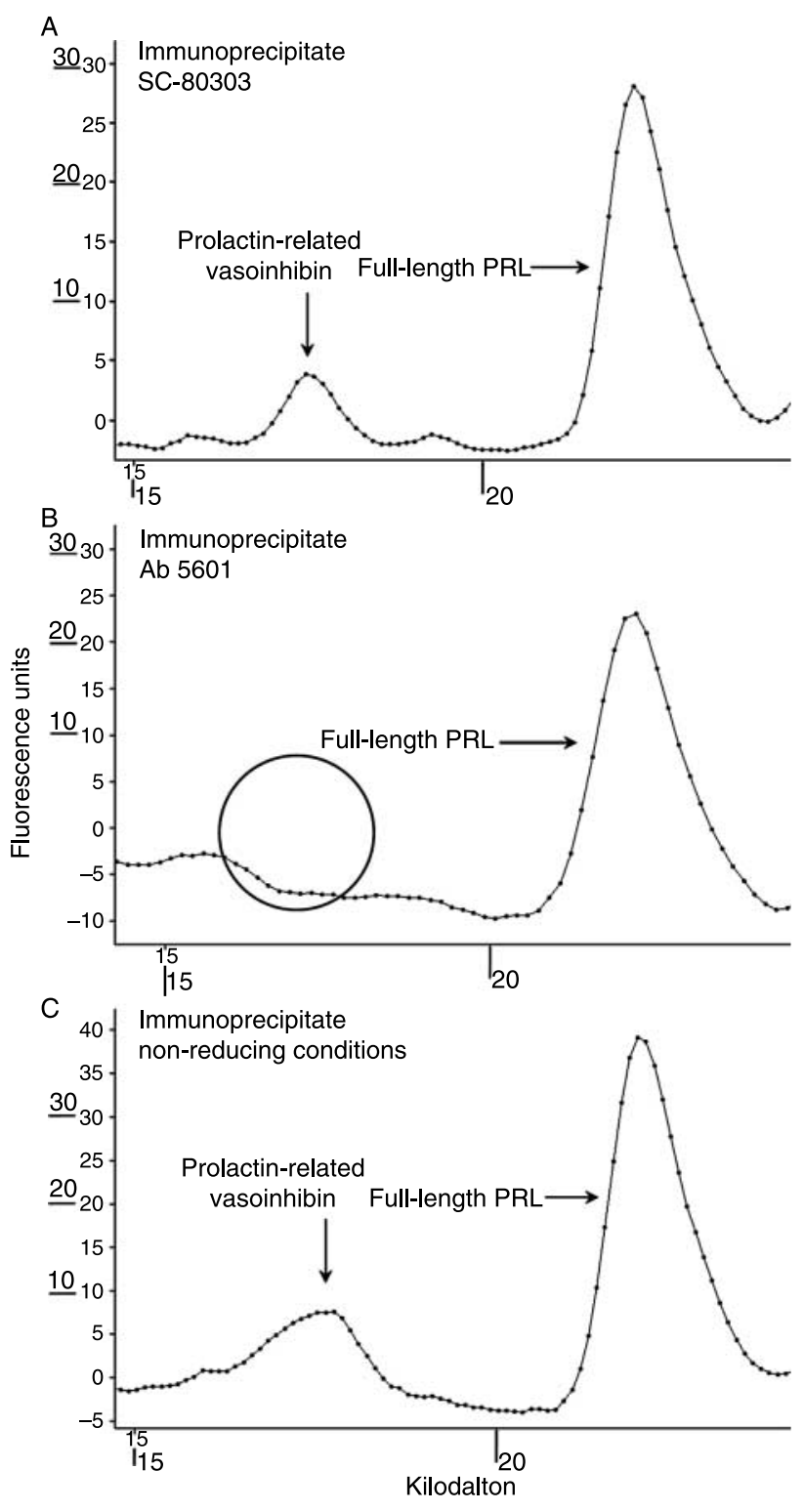

Figure 2 (A) Electropherogram of a serum-sample, immunoprecipitated with a different mouse MAB specific to hPRL, SC-80303, confirms prolactin-related vasoinhibin and $23 \mathrm{kDa}-\mathrm{PRL}$ signals. (B) Negative control. Analysis of a serum-sample, immunoprecipitated with a mouse MAB, specific to an epitope at the $C$-terminal end of PRL, Ab 5601, shows a $23 \mathrm{kDa}$-PRL, but no prolactin-related vasoinhibin signal. (C) Analysis of a serum-sample immunoprecipitated with the antibody 5602 under non-reducing conditions, demonstrates, compared with analysis under reducing conditions, a similar pattern.

Carpinteria, CA, USA) was added. After $3 \mathrm{~h}$ at $4{ }^{\circ} \mathrm{C}$ under rotation, $50 \mu \mathrm{l}$ protein $\mathrm{A}$-agarose was added and the sample was incubated for $12 \mathrm{~h}$. Following centrifugation $(20 \mathrm{~s}$ at $3800 \boldsymbol{g})$, the supernatant was discharged and the pellet was washed thrice in PBS. The pellet was then resuspended in $30 \mu \mathrm{l}$ SDS-PAGE loading buffer containing $\beta$-mercaptoethanol, boiled for 
$5 \mathrm{~min}$ and then left on ice for $5 \mathrm{~min}$ to cool down. After centrifugation $(20 \mathrm{~s}$ at $3800 \boldsymbol{g})$, the supernatant was electrophoresed on a $17 \%$ SDS-PAGE and an electroblot was performed on a PVDF membrane. The amersham low-range rainbow molecular weight marker (3500-40 $000 \mathrm{kDa}$, product code: RPN755E) has been used to determine the molecular weight. The transfer was conducted semi-dry at $0.8 \mathrm{~mA} / \mathrm{cm}^{2}$. The membrane was blocked for $12 \mathrm{~h}$ with tris buffered saline (TBS) containing $8 \%$ dry milk. Next, the membrane was incubated with a 1:500 dilution of another rabbit polyclonal antibody against hPRL (Cat. No.: Ab1971, Abcam, Cambridge, MA, USA) for $1 \mathrm{~h}$ at $37^{\circ} \mathrm{C}$ and afterwards with a 1:1000 dilution of a peroxidaseconjugated swine anti-rabbit antibody (Cat. No.: P0399, DakoCytomation). ECL western blotting reagents (GE Healthcare, Freiburg, Germany) were used to visualize the immunocomplexes (Fig. 3).

\section{Subjects}

The case group consisted of 21 patients with diabetes mellitus with either proliferative $(n=14)$ or nonproliferative $(n=7)$ diabetic retinopathy, assigned to a respective subgroup. For the control group, 27 healthy control subjects with no history of diabetes mellitus were enrolled. Patients of the case group were recruited at the Eye Clinic of the University of Goettingen. Ophthalmologic diagnosis was acquired through indirect ophthalmoscopy according to the criteria of the Early Treatment Diabetic Retinopathy Study (15). All control subjects were ambulant patients, recruited in a practice for internal medicine in Bochum, Germany. Institutional ethics committee approval and written informed consent from all participants were obtained.

Exclusion criteria for all patients were medications and conditions known to increase or decrease PRL levels: a medical history of prolactinoma, hypothyroidism, chronic renal failure, liver cirrhosis, lesions of the chest wall during the last 3 months before recruitment,

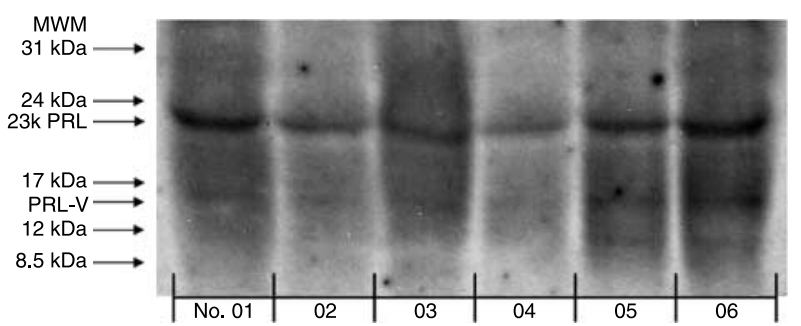

Figure 3 Western blot analysis. Immunoprecipitation of serum samples followed by western blot analysis under reducing conditions revealed clear $23 \mathrm{kDa}-\mathrm{PRL}$ (23K PRL) but only weak and non-reproducible prolactin-related vasoinhibin (PRL-V) bands. Samples 1-3: serum from patients with proliferative diabetic retinopathy, samples 4-6: samples from healthy controls. MWM, molecular weight marker. and treatment with cimetidine, cyproheptadine, monoamine oxidase (MAO)-inhibitors, meprobamate, methyldopa, metoclopramide, antipsychotic drugs, opiate, estrogen, prostaglandin, reserpine, sulpiride, tricyclic antidepressants, or verapamil. Blood samples were drawn from the cubital vein between 0600 and $1200 \mathrm{~h}$ and underwent screening for full-length PRL and 16K PRL.

\section{Statistical analysis}

Descriptive statistics were used to report the demographic and clinical characteristics of the study population. Age and years since diagnosis of the patients is presented as means in years \pm s.D. PRL concentrations are presented as means and their s.E.M. The signals of 16K PRL were read by signal strength, defined as peak height in fluorescence units (FU) shown in the electropherograms of the Bioanalyzer 2100 device. The Wilcoxon-Mann-Whitney test was used to determine significant differences. Differences in means with a $P$ value $<0.05$ were considered statistically significant. Statistical analysis performed by Prism 4 GraphPad Software (GraphPad Software, La Jolla, CA, USA).

\section{Results}

\section{Immunocomplex constituent analysis}

To identify the signals of the immunoprecipitate measurement and to evaluate the characteristics of each component in the Bioanalyzer 2100, we conducted an immunocomplex constituent analysis. Protein G-agarose, the affinity chromatography matrix used for the immunoprecipitation, was found to cause no peaks within the detection area. Analysis of the mouse MAB (anti-hPRL antibody 5602) under reducing conditions showed migration of the IgG light chains corresponding to a molecular weight of $\sim 26-28 \mathrm{kDa}$ and the heavy chain at $\sim 65 \mathrm{kDa}$ (Fig. 4), thereby not interfering with PRL signals. rhPRL was determined to migrate at $\sim 22 \mathrm{kDa}$ (Fig. 1A). Cathepsin D-cleaved PRL-fragments were determined to migrate at $11,15,16.5$, and $17 \mathrm{kDa}$ (Fig. 1B).

\section{Signal identification of the immunocomplex measurement}

Epitope mapping of the MAB 5602 by Piwnica et al. (5) demonstrates that this antibody binds to the N-terminal residues 1-9 of human PRL. It does not detect $\Delta$ 1-9-hPRL (lacking the nine N-terminal residues). The immunoprecipitation is specific to $\mathrm{N}$-terminal and full-length PRL but not C-terminal fragments. Thus, the $16-17 \mathrm{kDa}$ component of the immunocomplex, consistent with positive and negative 


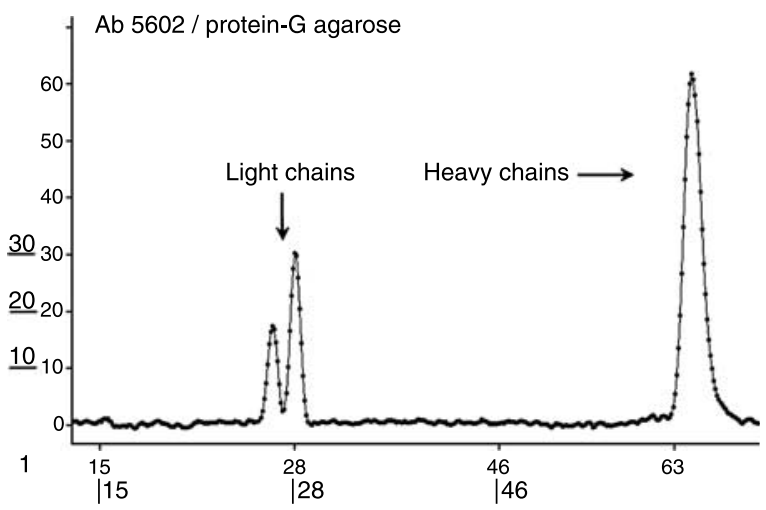

Figure 4 Electropherogram of the immunocomplex constituent analysis. The mouse MAB 5602 and protein-G agarose were analyzed under reducing conditions. The light chains migrate at a molecular weight of $\sim 26-28 \mathrm{kDa}$ and the heavy chain at $\sim 65 \mathrm{kDa}$. Protein-G agarose causes no peaks.

controls, was identified to represent N-terminal PRL-V and not a C-terminal PRL-fragment (Fig. 1C). In accordance with rhPRL as a positive control, we identified the signal at $22 \mathrm{kDa}$ to represent full-length PRL (Fig. 1C). A western blot analysis of the immunoprecipitates, using a rabbit polyclonal antibody against human PRL (A0569, DakoCytomation), also confirmed the identity of the $22 \mathrm{kDa}$-peaks but did not show any immunoreactive bands at 16-17 kDa (data not shown). Immunoprecipitation with another mouse MAB (SC-80303), binding to a different epitope on hPRL, and successive measurement of the immunoprecipitate according to the method described above, confirmed PRL-V and full-length PRL signals (Fig. 2A). This antibody binds to an epitope corresponding to amino acids 29-151. For the negative control, the immunoprecipitation was conducted with a mouse MAB against hPRL with an epitope at the C-terminal end of PRL (Ab 5601). Analysis of a sample previously shown to contain PRL-V by immunoprecipitation with two monoclonal antibodies with N-terminal epitopes (CAN-hPRL-4100-12, DBC; SC-80303, SCB) and subsequent analysis with the Bioanalyzer 2100 revealed a 23K PRL but no PRL-V signal (Fig. 2B).

\section{Analysis of PRL-V under non-reducing conditions}

Measurement of the immunoprecipitate under nonreducing conditions revealed, compared with analysis under reducing conditions, a similar pattern (Fig. 2C).

\section{Generation of PRL fragments}

Incubation of rhPRL with cathepsin D resulted in a proteolytic cleavage of full-length PRL into an 11, 15, 16.5 and $17 \mathrm{kDa}$ PRL fragment (Fig. 1C).

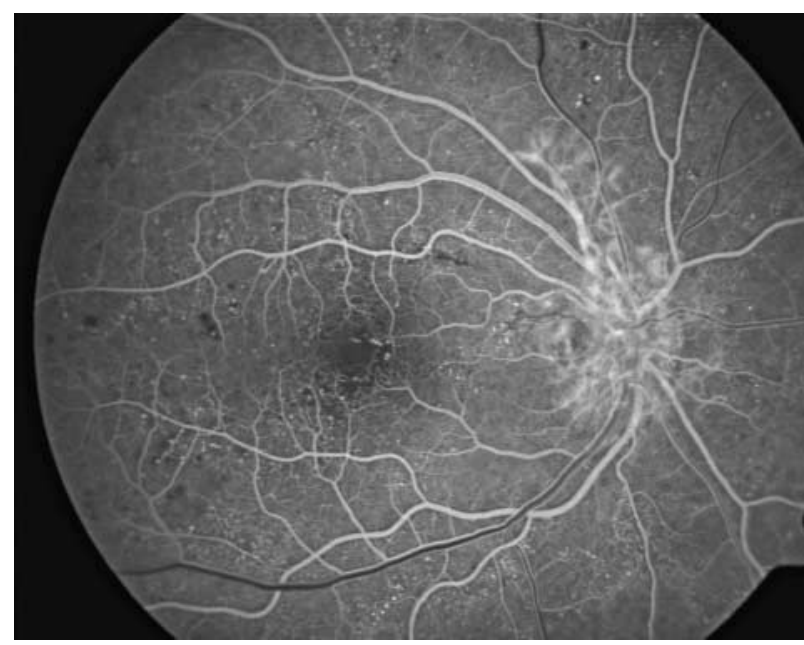

Figure 5 Fluorescein angiography of the retina of a patient from the case group diagnosed with proliferative retinopathy. Neovascularization and leaking vessels are seen around the papilla nervi optici.

\section{Demographic and clinical characteristics of the study population}

The patients in the case group had a mean age of $64.3 \pm 11.8$ years. The mean duration since the diagnosis of diabetes mellitus (Type I or II) was 19.6 \pm 10.0 years. Two subjects $(10 \%)$ in the case group had Type I diabetes mellitus and 19 (90\%) Type II diabetes mellitus. A total of 17 individuals $(80 \%)$ were treated with insulin and $4(20 \%)$ received oral hypoglycemic medication. A total of 14 patients $(66 \%)$ had proliferative diabetic retinopathy (Fig. 5) and 7 (33\%) non-proliferative diabetic retinopathy. The mean

Table 1 Demographic and clinical characteristics of the study population. Data are presented as number of individuals or mean years \pm s.D.

\begin{tabular}{lll}
\hline Characteristic & $\begin{array}{c}\text { Case group } \\
(n=21)\end{array}$ & $\begin{array}{c}\text { Control group } \\
(n=27)\end{array}$ \\
\hline Male sex $(n)$ & 21 & 27 \\
Age $($ year $)$ & $64.3 \pm 11.6$ & $54.0 \pm 18.3$ \\
Race $(n)$ & 20 & 26 \\
White & 0 & 1 \\
Black & 1 & 0 \\
Asian & 0 & 0 \\
Other & & \\
Diabetes type $(n)$ & 2 & \\
$\quad$ Type I & 19 & \\
$\quad$ Type II & $19.6 \pm 10.0$ & \\
Years since diagnosis of DM & & \\
Diabetes therapy $(n)$ & 4 & \\
$\quad$ Oral medication & 17 & \\
Insulin & & \\
Ophthalmologic diagnosis $(n)$ & & \\
RDP & 14 & \\
RDS & 7 &
\end{tabular}

DM, diabetes mellitus; RDP, retinopathia diabetica proliferans; RDS, retinopathia diabetica simplex. 


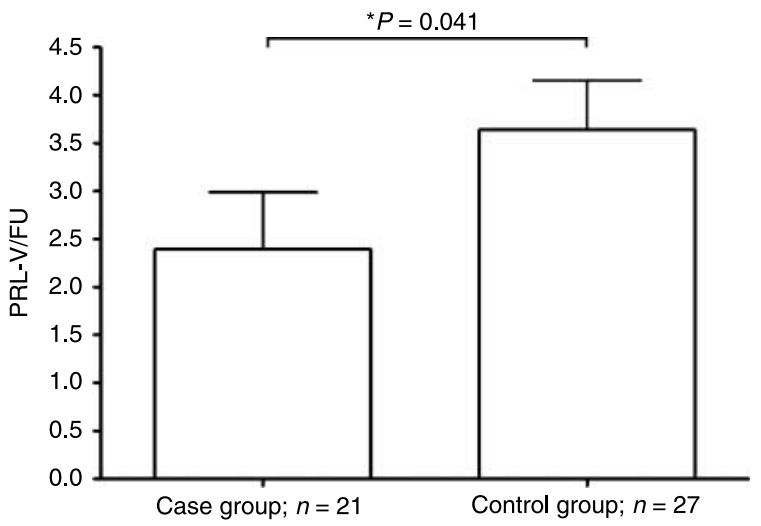

Figure 6 Serum prolactin-related vasoinhibin concentrations. The bars represent prolactin-related vasoinhibin (PRL-V) signals in fluorescence units (FU), mean \pm S.E.M., disclosed by immunoprecipitation of serum samples and subsequent analysis with the Bioanalyzer 2100. The case group had significantly lower prolactinrelated vasoinhibin concentrations than the control group $(P=0.041)$. The case group had a mean prolactin-related vasoinhibin concentration of $2.39 \pm 0.59 \mathrm{FU}$, whereas the control group had a concentration of $3.64 \pm 0.51 \mathrm{FU}$.

age of the patients in the control group was $54.0 \pm 18.3$ years. The demographic and clinical characteristics of the study population are summarized in Table 1.

\section{Serum PRL-V and 23K PRL concentrations}

Statistical analysis revealed that the case group had significantly lower PRL-V concentrations than the control group $(P=0.041)$. The case group had a mean PRL-V concentration of $2.39 \pm 0.59 \mathrm{FU}$, whereas the control group had a concentration of $3.64 \pm 0.51$ FU (Fig. 6, Table 2). Subgroup analysis showed no significant difference between either of the subgroups. Patients with proliferative diabetic retinopathy had a mean PRL-V concentration of $2.51 \pm 0.85 \mathrm{FU}$ and patients with non-proliferative diabetic retinopathy a mean PRL-V concentration of $2.15 \pm 0.56$ FU (Table 2). Figure 7 demonstrates representative electropherograms of a patient of the control group (Fig. 7A), a patient with retinopathia diabetica simplex (Fig. 7B) and a patient with retinopathia diabetica proliferans (Fig. 7C). Comparison of 23K PRL serum concentrations, measured by ECLIA, revealed no significant difference between either of the groups. The patients of the case group had a full-length PRL concentration of $238.6 \pm 19.38 \mathrm{mU} / \mathrm{l}$; analysis of the control group revealed a concentration of $222.1 \pm 19.02 \mathrm{mU} / \mathrm{l}$ $(P>0.05)$. Subgroup analysis of the case group showed that patients with proliferative diabetic retinopathy had a concentration of $233.5 \pm 26.20 \mathrm{mU} / \mathrm{l}$ and those with non-proliferative diabetic retinopathy had a concentration of $248.9 \pm 27.49 \mathrm{mU} / \mathrm{l}(P>0.05$; Table 2). Western blot analysis showed reproducible 23K PRL bands in all serum samples, but only weak, nonreproducible bands at $16 \mathrm{kDa}$, which were not sufficient for densitometric analysis (Fig. 3).

\section{Discussion}

It is a widely accepted model in the understanding of angiogenesis that neovascularization, in health and disease, is controlled by pro- and anti-angiogenic factors $(16,17)$. There has been an ongoing effort during the last decades to identify these factors and investigate their relevance in pathophysiological conditions (18). PRL-V has been identified as a natural inhibitor of angiogenesis in the retina (12), by inhibiting VEGF-mediated retinal vasopermeability (13) and antagonizing proangiogenic effects of VEGF (8). Angiogenesis and increased vasopermeability of retinal vessels are pathologic processes occurring in diabetic retinopathy. In this context, a role of PRL-V in the pathogenesis of diabetic retinopathy in humans is being discussed $(10,14)$. The present study supports the theory and provides evidence that patients with diabetic retinopathy have, compared with healthy subjects, lower levels of PRL-V in circulating blood. In view of the fact that PRL-V has antiangiogenic properties and a preventive effect on retinal vasopermeability, the lower concentration of PRL-V could contribute to the development and progression of diabetic retinopathy. Our results corroborate the

Table 2 Serum prolactin concentrations. The values are presented as means \pm S.E.M. Serum prolactin (PRL) concentrations were determined with a commercial electro-chemiluminescence immunoassay. Serum PRL-related vasoinhibin (PRL-V) concentrations were determined by immunoprecipitation of serum samples and subsequent measurement with the Bioanalyzer 2100 . Statistical analysis with the Wilcoxon-Mann-Whitney test revealed that the case group had significantly lower prolactin-related vasoinhibin concentrations than the control group $(P=0.041)$.

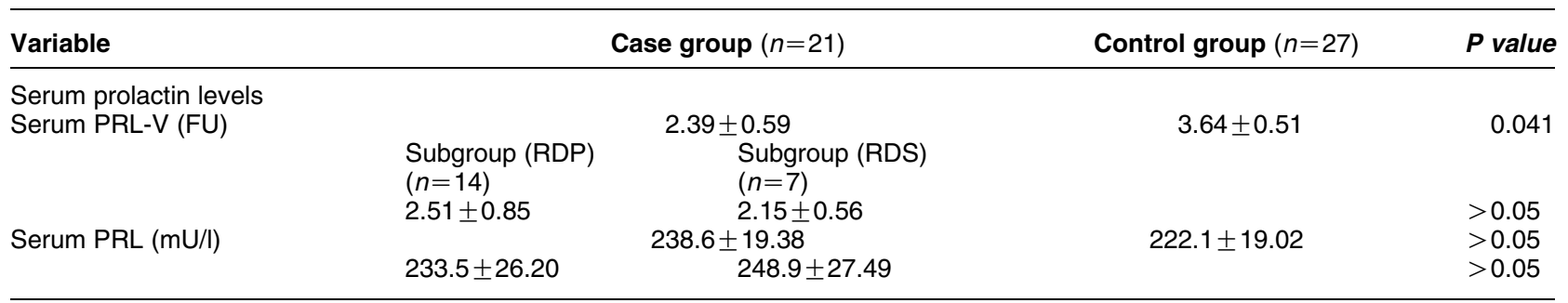

$\mathrm{RDP}$, retinopathia diabetica proliferans; RDS, retinopathia diabetica simplex. 


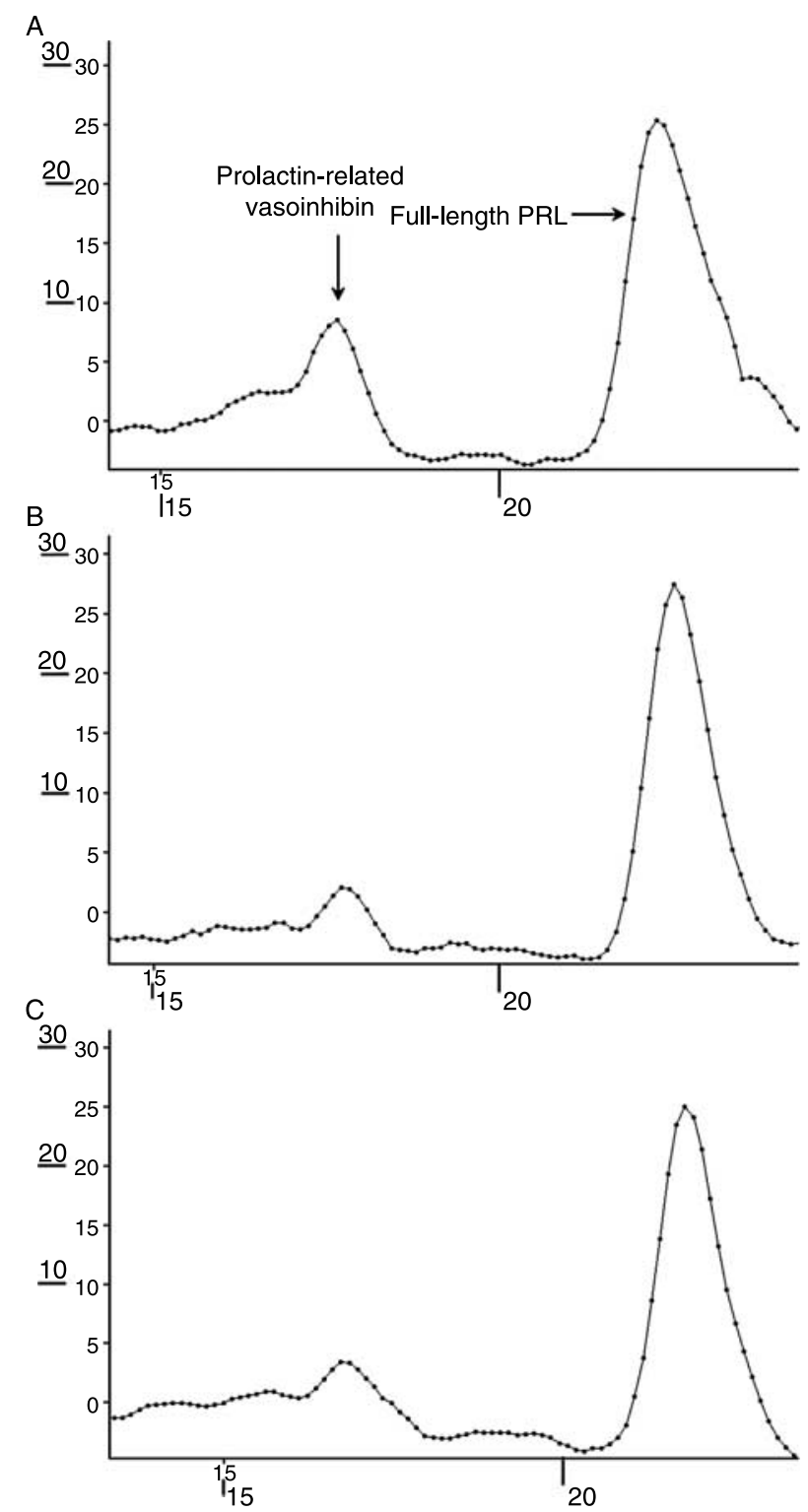

Figure 7 Representative electropherograms of a patient of the control group $(A)$ a patient with retinopathia diabetica simplex (B) and a patient with retinopathia diabetica proliferans (C).

assumption that pathological neovascularization in the eyes of patients with diabetes mellitus could be based on a systemic shift in the equilibrium of pro- and antiangiogenic factors in favor of enhanced angiogenic potential.

Several studies show that patients with diabetes mellitus have higher levels of VEGF in serum and vitreous fluid (19). Since PRL-V can inhibit VEGF-stimulated endothelial cell proliferation $(8,20)$, the lower levels of PRL-V could result in decreased VEGF-inhibition, thereby contributing to a pro-angiogenic environment.

Investigating patients with preeclampsia, LeañosMiranda et al. reported that patients with acute renal failure exhibited higher urinary PRL concentrations and higher urinary frequencies of antiangiogenic PRLfragments than patients without diminished renal function (21). Since impaired renal function is common in patients with diabetes mellitus, increased renal elimination could explain lower systemic concentrations of PRL-V. According to Ben-Jonathan et al. glycosylation may alter proteolytic cleavage of PRL (22). This may result in the decreased proteolytic production of PRL-V, and thus enhanced glycosylation due to hyperglycemia could explain decreased levels of PRL-V.

Besides a small study population, there are other limitations to our investigation. Clearly, as the blood samples were collected from the cubital veins, we cannot state any information of PRL-V distribution in the eye, e.g. ocular fluid. Nonetheless, systemic PRL can enter the eye (23) and potentially act in concert with, or in addition to, local factors. Although PRL is expressed by retinal tissues and PRL-V has been detected in the rat retina (24), the origin of ocular PRL could also be systemic. Furthermore, most of the information about PRL-V comes from either in vitro studies or in vivo studies on rodents. However, the antiangiogenic effects of PRL-V have been demonstrated for both rat and human PRL. Moreover, the variability in posttranslational modifications between PRL from these species is not substantial (22).

Since, according to the manufacturer (Agilent Technologies) of the device Agilent 2100 Bioanalyzer, the sizing resolution is $10 \%$ and the sizing accuracy has a $10 \%$ coefficient of variation, we cannot discriminate between 16, 16.5, and $17 \mathrm{kDa}$ PRL. To our knowledge, there is currently no method or assay available, which is capable of isolating, semi-quantitatively measuring, and discriminating between 16,16.5, and $17 \mathrm{kDa}$ PRL.

Measurement of the immunoprecipitate under nonreducing conditions revealed, compared with analysis under reducing conditions, a similar pattern (Fig. 2C). Several measurements with and without the reducing agent ( $\beta$-mercaptoethanol) demonstrate that it does not affect the immunocomplexes. When using the Agilent 2100 Bioanalyzer, the addition of the reducing agent does not change or improve the migration properties. We think that similar results under reducing and nonreducing condition show that the addition of a reducing agent does not introduce laboratory-made artifacts.

The western blot analysis showed reproducible $23 \mathrm{~K}$ PRL bands in all samples, but only weak, nonreproducible bands at $16 \mathrm{kDa}$, which were not sufficient for densitometric analysis (Fig. 3). The two immunoreactive bands correlate close to the $24 \mathrm{kDa}$ band and the $17 \mathrm{kDa}$ band of the marker, consistent with fulllength PRL and PRL-V. In addition to Ab1971, we used the MAB mAb 5602 and the polyclonal antibody A0569 in multiple western blots, attempting to detect PRL-V. Both antibodies repeatedly and reproducibly detected full-length PRL but failed to show immunoreactive bands at $16 \mathrm{kDa}$. Ab1971 was the only 
antibody showing immunoreactive bands at $16 \mathrm{kDa}$, but even this signal was not reproducible and therefore not suitable for a quantitative analysis (optical densitometry). A reproducible qualitative and quantitative analysis of PRL-V from human serum by immunoprecipitation and subsequent western blot has not been described to our knowledge. We suspect the major reason for this is the extremely low amount of antigen. We recognized this by unsuccessfully attempting multiple western blots with dozens of variations in order to detect PRL-V, and subsequently started to combine immunoprecipitation with the laser-induced fluorescence technique instead of western blot.

PRL-V has been linked to the pregnancy-associated diseases post partum cardiomyopathy (25) and preeclampsia (21). In our investigation, the detection of PRL-V in serum samples with immunoprecipitation procedures followed by western blot analysis was either unsuccessful or not reproducible. In the study by Hilfiker-Kleiner et al. (25), the detection of PRL-V in serum samples by western blot analysis was only successful in samples from lactating women who have, due to its function in milk production and lactation, high systemic PRL levels. In our study, we detected PRL-V in a series of serum samples from male subjects with full-length PRL levels within the normal range. Therefore, we believe that the use of immunoprecipitation in combination with laser induced fluorescence is a new technique, which could facilitate further research in this important field.

This study aimed to show whether PRL-V serum levels correlate with the presence of diabetic retinopathy. In this context, we chose a control group of healthy subjects. A control group of patients with diabetes mellitus but no microvascular complications was considered inferior since a monocausal role of PRL-V in the development and progression of diabetic retinopathy is unlikely. However, since we found decreased levels of PRL- $V$ in patients with diabetic retinopathy, the investigation of PRL-V levels in diabetic patients without microvascular complications is an important question, which requires further research.

In summary, we show that patients with diabetes mellitus and diabetic retinopathy have, compared with healthy subjects, decreased serum levels of PRL-V in systemic blood. In view of the antiangiogenic and antivasopermeability actions of PRL-V, we conclude that the decreased vasoinhibin serum levels could contribute to the development and progression of diabetic retinopathy. Future investigations, disclosing PRL-V levels in patients with diabetes mellitus and no microvascular complications, will help to further evaluate the role of PRL-V in diabetic retinopathy.

\section{Declaration of interest}

The authors state that they have no conflict of interest to declare.

\section{Funding}

This research did not receive any specific grant from any funding agency in the public, commercial, or not-for-profit sector.

\section{Acknowledgements}

This study was conducted in cooperation with the Eye Clinic of the University of Göttingen. We gratefully acknowledge our colleague Dr $\mathrm{M}$ Tondrow for his expert opinion and support with patient recruitment. We thank PhD J Dudas from our department for excellent technical assistance and D Suan MD for the critical review of the manuscript.

\section{References}

1 Klein R, Klein BEK, Moss SE, Davis MD \& DeMets DL. Glycosylated hemoglobin predicts the incidence and progression of diabetic retinopathy. Journal of the American Medical Association 1988260 2864-2871.

2 Frank RN. Diabetic retinopathy. New England Journal of Medicine $200435048-58$.

3 Clapp C, López-Gómez FJ, Nava G, Corbacho A, Torner L, Macetola Y, Duenas Z, Ochoa A, Noris G, Acosta E, Garay E \& Martínez de la Escalera G. Expression of prolactin mRNA and of prolactin-like proteins in endothelial cells: evidence for autocrine effects. Journal of Endocrinology 1998158 137-144.

4 Ben-Jonathan N, Mershon JL, Allen DL \& Steinmetz RW. Extrapituitary prolactin: distribution, regulation, functions, and clinical aspects. Endocrine Reviews 199617 639-669.

5 Sinha YN. Structural variants of prolactin: occurrence and physiological significance. Endocrine Reviews 199516 354-369.

6 Piwnica D, Touraine P, Struman I, Tabruyn S, Bolbach G, Clapp C, Martial JA, Kelly PA \& Goffin V. Cathepsin D processes human prolactin into multiple $16 \mathrm{~K}$-like N-terminal fragments: study of their antiangiogenetic properties and physiological relevance. Molecular Endocrinolgy 200418 2522-2542.

7 Khurana S, Liby K, Buckley AR \& Ben-Jonathan N. Proteolysis of human prolactin: resistance to cathepsin $\mathrm{D}$ and formation of a nonangiostatic, C-terminal $16 \mathrm{~K}$ fragment by thrombin. Endocrinology 1999140 4127-4132.

8 Clapp C, Martial JA, Guzman RC, Rentier-Delrue F \& Weiner RI. The 16-kilodalton N-terminal fragment of human prolactin is a potent inhibitor of angiogenesis. Endocrinology 1993133 1292-1299.

9 Struman I, Bentzien F, Lee H, Mainfroid V, D’Angelo G, Goffin V, Weiner RI \& Martial JA. Opposing actions of intact and N-terminal fragments of the human prolactin/growth hormone family members on angiogenesis: an efficient mechanism for the regulation of angiogenesis. PNAS 199996 1246-1251.

10 Clapp C, Aranda J, Gonzalez C, Jeziorski MC \& Martínez de la Escalera G. Vasoinhibins: endogenous regulators of angiogenesis and vascular function. Trends in Endocrinology and Metabolism 200617 301-307.

11 Corbacho AM, Martínez de la Escalera G \& Clapp C. Roles of prolactin and related members of the prolactin/growth hormone/placental lactogen family in angiogenesis. Journal of Endocrinology $2002173219-238$.

12 Aranda J, Rivera JC, Jeziorsky MC, Riesgo-Escovar J, Nava G, López-Barrera F, Quiróz-Mercado H, Berger P, Martínez de la Escalera G \& Clapp C. Prolactins are natural inhibitors of angiogenesis in the retina. Investigative Ophthalmology and Visual Science $2005462947-2953$.

13 Garcia C, Aranda J, Arnold E, Thébault S, Macetola Y, LópezCasillas F, Mendoza V, Quiróz-Mercado H, Hernández-Montiel LH, Lin SH, Martínez de la Escalera G \& Clapp C. Vasoinhibins prevent 
retinal vasopermeability associated with diabetic retinopathy in rats via protein phosphatase $2 \mathrm{~A}$-dependent eNOS inactivation. Journal of Clinical Investigation $2008 \mathbf{1 1 8} 2291-2300$.

14 Clapp C, Thebault S, Arnold E, Garcia C, Rivera JC \& Martínez de la Escalera G. Vasoinhibins: novel inhibitors of ocular angiogenesis. American Journal of Physiology. Endocrinology and Metabolism 2008 295 E772-E778.

15 Early Treatment Diabetic Retinopathy Study Research Group. Results from the early treatment diabetic retinopathy study. Ophthalmology 199198 739-840.

16 Folkman J. Angiogenesis in cancer, vascular, rheumatoid and other disease. Nature Medicine 19951 27-31.

17 Folkman J \& Klagsburn M. Angiogenic factors. Science 1987325 442-447.

18 Nyberg P, Xie L \& Kalluri R. Endogenous inhibitors of angiogenesis. Cancer Research $2005653967-3979$.

19 Maier R, Weger M, Haller-Schober EM, El-Shabrawi Y, Wedrich A, Theisl A, Aigner R, Barth A \& Haas A. Multiplex bead analysis of vitreous and serum concentrations of inflammatory and proangiogenic factors in diabetic patients. Molecular Vision $2008 \mathbf{1 4}$ 637-643.

20 Caldwell RB, Bartoli M, Behzadian MA, El-Remessy AEB, Al-Shabrawey M, Platt DH \& Caldwell RW. Vascular endothelial growth factor and diabetic retinopathy: pathophysiological mechanisms and treatment perspectives. Diabetes/Metabolism Research and Reviews $200319442-455$.

21 Leanos-Miranda A, Marquez-Acosta J, Cardenas-Mondragon GM, Chinolla-Arellano ZL, Rivera-Leanos R \& Bermejo-Huerta S.
Urinary prolactin as a reliable marker for preeclampsia, its severity, and the occurrence of adverse pregnancy outcomes. Journal of Clinical Endocrinology and Metabolism 2008 93 2492-2499.

22 Ben-Jonathan N, LaPensee CR \& LaPensee EW. What can we learn from rodents about prolactin in humans? Endocrine Reviews 2008 29 1-41.

23 O'Steen WK \& Sundberg DK. Patterns of radioactivity in the eyes of rats after injections of iodinated prolactin. Ophthalmic Research $19821454-62$.

24 Ochoa A, Montes de Oca P, Rivera JC, Duenas Z, Nava G, Martínez de la Escalera G \& Clapp C. Expression of prolactin gene and secretion of prolactin by rat retinal capillary endothelial cells. Investigative Ophthalmology and Visual Science $2001 \mathbf{4 2}$ 1639-1645.

25 Hilfiker-Kleiner D, Kaminski K, Podewski E, Bonda T, Schaefer A, Sliwa K, Forster O, Quint A, Landmesser U, Doerries C, Luchtefeld M, Poli V, Schneider MD, Balligand JL, Desjardins F, Ansari A, Struman I, Nguyen NQN, Zschemisch NH, Klein G, Heusch G, Schulz R, Hilfiker A \& Drexler H. A cathepsin D-cleaved $16 \mathrm{kDa}$ form of prolactin mediates post partum cardiomyopathy. Cell $2007 \mathbf{1 2 8}$ 589-600.

Received 10 May 2009

Accepted 27 May 2009 